\title{
Clinical Study Site Accrual Status
}

National Cancer Institute

\section{Source}

National Cancer Institute. Clinical Study Site Accrual Status. NCI Thesaurus. Code C70785.

Defines the status of a clinical study site in relation to the subject enrollment at a particular time. 